\title{
Tracing the evolution of the two energy gaps in magnesium diboride under pressure
}

\author{
V. Kononenko ${ }^{1}$, V. Tarenkov ${ }^{1}$, M. Belogolovskii ${ }^{1,2}$, S. Döring ${ }^{3}$, \\ S. Schmidt ${ }^{3}$, and P. Seidel ${ }^{3}$ \\ ${ }^{1}$ A. Galkin Donetsk Institute for Physics and Engineering, NASU, Kyiv UA-03680, Ukraine \\ E-mail: bel@fti.dn.ua \\ ${ }^{2}$ G.V. Kurdyumov Institute for Metal Physics, Kyiv UA-03680, Ukraine \\ E-mail: belogolovskii@ukr.net \\ ${ }^{3}$ Institut für Festkörperphysik, Friedrich-Schiller-Universität Jena, Jena D-07743, Germany \\ E-mail: Paul.Seidel@uni-jena.de
}

Received November 17, 2014, revised December 18, 2014,

published online February 23, 2015

\begin{abstract}
We have studied transport characteristics of mesoscopic multiple-mode superconducting contacts formed between two grains in bulk two-gap magnesium diboride. The experimental setup was realized by driving a normal-metal tip into $\mathrm{MgB}_{2}$ polycrystalline sample and proved to be extremely stable, providing possibility to perform pressure experiments at low temperatures. It is argued that in our procedure a small piece of the superconducting electrode is captured by the tip apex and, as a result, two junctions in series are formed: a junction between a tip and $\mathrm{MgB}_{2}$ grain and a mesoscopic disordered contact between two superconducting pellets. Although the relative weight of the first junction resistance was considerably less, its contribution is shown to be important for the comparison of measured data with expected gap values. Two hallmarks of multiple Andreev reflections inside the $\mathrm{MgB}_{2}-\mathrm{c}-\mathrm{MgB}_{2}$ contact (c stands for a high-transparent constriction), a zero-bias $1 / \sqrt{|V|}$-like singularity of the dc differential conductance and peaks connected to the two gap values, have been revealed. Finally, we report results of a hydrostatic compression experiment showing the evolution of the $\mathrm{MgB}_{2}$ gap values with pressure. In contrast to the theoretical expectations, we have observed an increase of the smaller gap $\Delta_{\pi}$ whereas the larger gap $\Delta_{\sigma}$ decreased with increasing pressure as it should be for the electron-phonon pairing mechanism. We argue that the so-called separable model of anisotropy effects is insufficient to describe such changes and only improved two-band versions are capable to reproduce the pressure effect on the energy gaps in magnesium diboride.
\end{abstract}

PACS: 74.70.Ad Metals; alloys and binary compounds (including A15, MgB2, etc.);

81.07.Lk Nanocontacts;

74.62.Fj Effects of pressure.

Keywords: magnesium diboride, two-band/two-gap superconductor, high-transparency junctions, differential conductance, hydrostatic pressure.

\section{Introduction}

Conventional BCS theory of phonon-mediated superconductivity considers a single-valued order parameter $\Delta=$ const in the studied materials. In general, it is not constant but a momentum- and frequency-dependent function $\Delta(\mathbf{k}, \omega)$ which can be determined using fully anisotropic Eliashberg equations with the electron-phonon interaction characteristic $\lambda\left(\mathbf{k}, \mathbf{k}^{\prime}, \omega\right)$ [1]. In some superconductors such as magnesium diboride, experimental studies of the super- conducting state reveal the presence of two separate gaps. Usually, their appearance is associated with the presence of two very different sheets of the Fermi surface with considerably weak interaction between them [2]. In the simplest version of the two-band model, each band is determined by an independent set of parameters, including constant values of the coupling strength $\lambda_{i}(i=1,2)$ and those of the Fermi velocity $v_{F, i}(i=1,2)$. Besides, the two-band approach provides two additional parameters $\lambda_{i j}(i \neq j)$, interband coupling strengths, which are independent on 
the $\lambda_{i}$ values and can be varied freely within the model. It is clear that the solely intraband pairing picture is not sufficient to describe the main characteristics of two-band superconductors with a single critical temperature $T_{c}$. To reproduce some of the experimental features like the ratio of large gap/small gap, it is necessary to consider both intraband and interband pairing channels on an equal footing [3].

As was emphasized in the recent review [4], an alternative approach based on the account of anisotropy effects, the so-called separable model, can be proposed and the discrimination of the two approaches is very difficult in most cases. Whereas in the two-band picture two distinct gaps arise from the presence of two well-discriminated Fermi bands, in the separable one it refers to the variation within one band. The simplest way for introducing the anisotropy into a single-band electron-phonon interaction characteristic is to assume that $\lambda\left(\mathbf{k}, \mathbf{k}^{\prime}, \omega\right)=\left(1+a_{\mathbf{k}}\right) \lambda(\omega)\left(1+a_{\mathbf{k}^{\prime}}\right)$ with $\lambda(\omega)$ being the Fermi surface average of $\lambda\left(\mathbf{k}, \mathbf{k}^{\prime}, \omega\right)$ [5]. An important difference between the models resides in the fact that in the latter case we have the only main parameter $\lambda(\omega)$ that defines the average values of two narrow gap sets (they may be roughly interpreted as two different gaps), as the large gap/small gap ratio. On the contrary, for the two-band model, we should discriminate between intraband and interband impurity scattering rates. Thus, the interpretation of corresponding experimental data should be carried out with caution in order to separate one approach from the other [4].

This task is rather complicated, in particular, for such spatially directed techniques like tunneling or point-contact since the total quasiparticle current in related heterostructures is a sum of partial contributions from electronic groups with different gap values $\Delta_{i}(i=1,2)$. The weighing factor is proportional to the corresponding squared plasma frequency $\omega_{p, i}^{2}$ [6] and the relative contribution of the electronic groups to the spectra of a two-gap superconductor can be strongly anisotropic in the case of an anisotropic Fermi surface. If so, an additional feature related to one of two gaps can be undetectable for certain transport directions. But even if the two gap features are observed in the point-contact or tunneling experiments it cannot be a decisive measurement for the two-band interpretation since the anisotropic single-band scenario cannot be completely excluded in this case [4]. The best way to understand whether it is sufficient to describe a two-gap superconductor with a single electron-phonon interaction characteristic $\lambda(\omega)$ (the separable model) or the interband coupling parameter being independent on the intraband ones (the two-band approach) would be measurements of the effect of a controllable external parameter on the energy-gap structure in a superconductor.

In this work we show that hydrostatic high-pressure experiments on magnesium diboride polycrystalline samples provide additional opportunities to do more quantitative and reliable study of the role of the interband coupling and new arguments in support of the two-band model. In the next section, we describe the studied $\mathrm{MgB}_{2}$ specimens, a pressure chamber, and a procedure to create superconductor (S)-constriction (c)-superconductor junction by pressing a normal-metal $(\mathrm{N})$ tip into a magnesium diboride microcrystal. In the third section, we analyze current-vs-voltage and differential conductance-vs-voltage characteristics of the $\mathrm{S}-\mathrm{C}-\mathrm{S}$ junctions at zero pressure. The fourth section presents the data obtained in high-pressure measurements that demonstrate the evolution of those two gaps in $\mathrm{MgB}_{2}$ with pressure. To our best knowledge, these are the first experiments on the pressure effect on the gap structure in $\mathrm{MgB}_{2}$. The main results of the work are summarized in the last section.

\section{Experimental}

An initial $\mathrm{MgB}_{2}$ powder was synthesized during six hours by high-temperature reaction between boron and magnesium powders taken in stoichiometric ratio and grinded properly. The sintering temperature was $\leq 800{ }^{\circ} \mathrm{C}$ and the residual pressure was $\sim 10^{-2} \mathrm{~Pa}$. The polycrystalline samples used in the present work were obtained by uniaxial compression of the $\mathrm{MgB}_{2}$ powder. Annealed copper wires were stacked on a polished carbide anvil parallel to one other. The distance between the wires and their thickness controlled spatial dimensions and geometry of the probed area. Next, the $\mathrm{MgB}_{2}$ powder was poured between the wires and covered by the upper anvil. Both anvils were pressed against each other resulting in 6-7 GPa of pressure. Under the pressure, the $\mathrm{MgB}_{2}$ powder was compacted into mechanically durable bars with typical dimensions about $10 \times 1.0 \times 0.1 \mathrm{~mm}$.

The current-vs-voltage $I-V$ characteristics and the differential conductance $G(V)=d I(V) / d V$-versus- $V$ spectra were measured with a standard four-probe method and a lock-in technique by superposing a small ac-modulation of $10 \mu \mathrm{V}$ on a slowly varying voltage bias $V$ applied to the sample. The dynamic conductance was determined through numerical differentiation as well. Both results were compared with each other.

Contact pads with resistances about $10^{-3} \Omega$ were made of a fine silver powder adding to corresponding $\mathrm{MgB}_{2}$ areas before the compression procedure. With decreasing temperature, at $T=T_{c} \approx 39 \mathrm{~K}$, the well-established critical temperature value of the bulk magnesium diboride, resistance of the specimens started to fall sharply but the transition to the entirely superconducting state always took place at least a few degrees below the onset temperature (see the main panel in Fig. 1). All measurements were performed well below $30 \mathrm{~K}$ when the entire volume of the material was in the superconducting state.

We believe that the comparatively wide resistive transitions of the $\mathrm{MgB}_{2}$ bars with the width of several $\mathrm{K}$ originate from their granular nature. It is well known that 


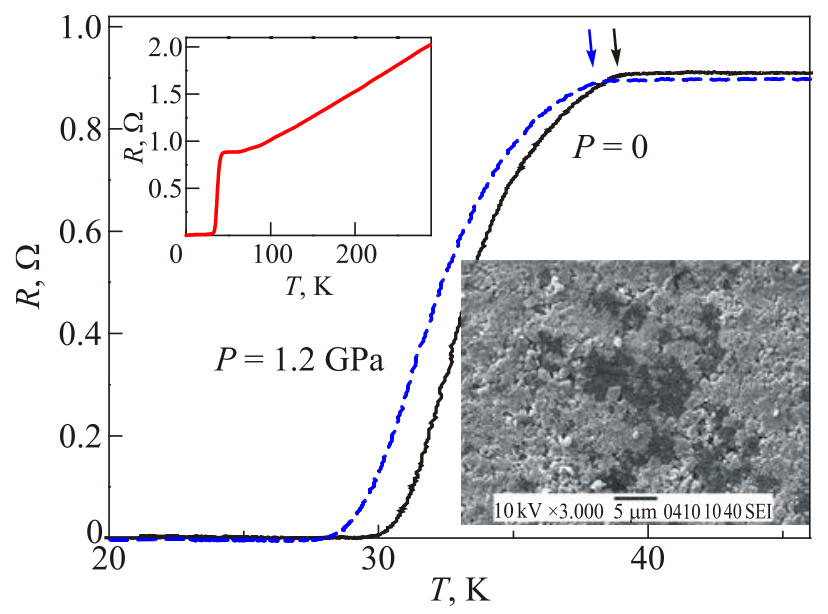

Fig. 1. Resistive normal-to-superconductor transition $R$ of the granular $\mathrm{MgB}_{2}$ sample at ambient pressure (the solid line) and under the pressure of $1.2 \mathrm{GPa}$ (the dashed line). The left inset demonstrates the resistance-vs-temperature dependence from $T=4.2 \mathrm{~K}$ to room temperature; the right inset shows a SEM image of a $\mathrm{MgB}_{2}$ sample.

granular metal composites are characterized by two distinct electronic transport regimes, percolation and tunneling ones, depending on the relative amount of the metallic phase and the nature of the grain interspace [7]. At sufficiently large volume fraction of the metallic phase, such samples behave as percolating systems with a well-defined critical concentration above which macroscopic clusters of physically connected conductive particles span the entire sample. This "metallic regime" is characterized by the resistivity increasing linearly with the temperature which is the case at $T>80 \mathrm{~K}$ (see the left inset in Fig. 1). Essentially granular nature of our $\mathrm{MgB}_{2}$ samples with well connected grains was confirmed by the scanning electron microscope JSM-6490LV analysis (see the right inset in Fig. 1). We show below that granularity of our $\mathrm{MgB}_{2}$ samples provides the possibility to create contacts between individual superconducting granules.

The experimental setup was prepared by the following way. A silver tip soldered to a spring made of beryllium bronze was attached to the end portion of the $\mathrm{MgB}_{2}$ bar welded to a glass fiber (Fig. 2). The contact resistance was determined by the spring pressing strength and thus could be varied in a very wide range. Note that in contrast to a similar procedure in the paper [8], we have not released the pressure after pushing the normal-metal tip into $\mathrm{MgB}_{2}$. In our opinion, it was the reason why we have observed formation of direct high-transparent inter-grain contacts while the authors [8] registered a typical tunneling behavior in the most cases.

As was declared in the introduction, the main driving force behind our experiment was to study the pressure influence on superconducting gaps in magnesium diboride. Because of that, we have paid special attention to the mechanical stability of the junctions. Upon reaching the opti-

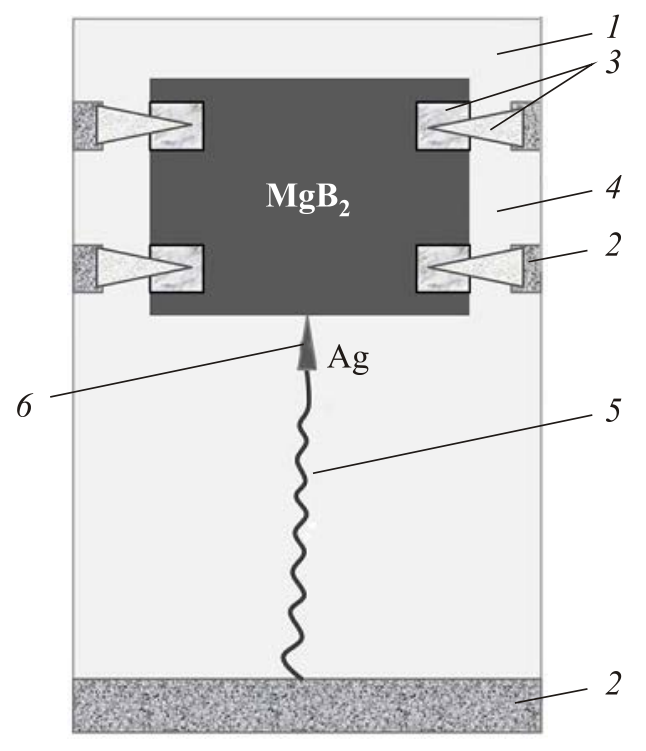

Fig. 2. Top view of the $\mathrm{Ag} / \mathrm{MgB}_{2}$ setup: substrate (1); $\mathrm{Ag}$ contact pads (2); Cu pads (3); $\mathrm{MgB}_{2}$ bar (4); spring (5); silver tip (6).

mal values of the contact resistance about several tens of Ohms the contact area was coated by a layer of the varnish. After its polymerization, the contact was rigidly fixed and the junctions demonstrated well reproduced characteristics after several heating and cooling cycles as well as pressure ones.

The high-pressure experiments were performed in a pressure chamber with a piston and kerosene oil as pressuretransmitting medium. 24 conductors were put into the chamber allowing us to place additionally a manganin high-pressure sensor and a platinum-wire thermometer. In the main panel of Fig. 1 we demonstrate the pressure effect on the normal-to-superconductor transition in our $\mathrm{MgB}_{2}$ samples. Decreasing rates of the onset temperature $T_{C}$ (shown by corresponding arrows) as well as those of the midpoints in resistance-vs- $T$ dependences were about $1 \mathrm{~K} / \mathrm{GPa}$ in good agreement with results by other authors for $T_{C}$ in $\mathrm{MgB}_{2}$ (see an overview of relevant experimental data in $[9,10]$ ). Thus, the relative change of both characteristics was about $0.031 / \mathrm{GPa}$ [9]. The normal-state resistance decrease was of the same order $\sim 0.02-0.031 / \mathrm{GPa}$.

We note that the same compression-chamber technique was successfully used by us earlier to study the pressure effect on the differential resistance characteristics of point contacts formed by a silver counter-electrode and high- $T_{C}$ cuprate ceramics in the superconducting state (see, e.g., $[11,12])$.

\section{Conductance spectra at ambient pressure}

Totally, at ambient pressure we have measured about 50 point contacts formed by a silver tip with magnesium diboride bars. All current-voltage $I(V)$ characteristics clearly exhibited an excess current proving the presence of a high- 
transparent superconducting junction. The overwhelming majority of the samples demonstrated also two prominent features in the $G(V)$ spectra: a strong zero-bias maximum with $G(V=0)$ which usually exceeded more than two times the normal-state value $G_{N} \approx G\left(e V>>\Delta_{\sigma}\right)$ as the limit for SN Andreev reflection transport and two small but well pronounced peaks at voltages slightly above 3 and $7 \mathrm{meV}$. The typical experimental dependence $G(V)$ is shown in Fig. 3(a), curve 1. Sometimes (only a few samples) we have observed an additional third peak at approximately $9 \mathrm{meV}$. The junction with the most prominent feature at $9 \mathrm{meV}$ (see Fig. 3(b), curve 1) was selected for subsequent pressure experiments.

From the first sight, the studied samples are expected to be contacts formed by a normal-metal tip, a superconducting grain, and a constriction c between them. In this welldeveloped point-contact technique, the metallic tip is brought into direct NS-contact which size should be smaller than the electron mean free path. If a direct contact between the counter-electrode and a single-band superconductor is established, for voltages $|V|<\Delta / e$, at zero temperature, only electron-into-hole and inverse scatterings known as Andreev-reflection processes take place, which double the differential interface conductance $G(V)$ with respect to the $G_{N}$ value. Only for $|V|>\Delta / e$ single electrons may propagate from the normal part of the contact to the superconducting one. As a result, the interface conductance drops to its normal value at $|V| \gg \Delta / e$. When a finite barrier is present at the interface, we should observe a reduction of the low-voltage conductance and a conductance peak at $|V|=\Delta / e$.

Our $d I / d V-v s-V$ data do not agree with these expectations (see Fig. 3). Instead of a step-like behavior around $|V|=\Delta_{\pi} / e$ and a nearly doubled value for voltage biases $|V|<\Delta_{\pi} / e$ [15], we have observed, first, a huge zero-bias conductance peak and, second, a set of nonlinear features in the energy-gap region. As to the first issue, there can be several explanations. The simplest one may be related to a superconducting quenching effect in a narrow microbridge between two metallic electrodes. As was argued in Ref. 16 and references therein, the quenching mechanisms can be classified in two classes: (a) electrodynamic fluxflow vortex effects and (b) heat-driven thermal instabilities. Both of them may be relevant in our experiments. Ignoring the presence of the zero-bias anomaly, we could interpret the two peaks in Fig. 3(a) as signatures of the two gaps $\Delta_{\pi}$ and $\Delta_{\sigma}$ in $\mathrm{MgB}_{2}$ and the third nonlinearity in Fig. 3(b) as that related to the sum $\Delta_{\pi}+\Delta_{\sigma}$. But following this assumption, the value of $\Delta_{\pi}$ would be overestimated and that of $\Delta_{\sigma}$ would be lower than generally accepted, e.g. $[14,15]$.

To solve this contradiction, we follow the papers $[8,17]$ and interpret the observation by assuming that our contacts are formed by two junctions in series, a point contact between the normal-metal tip and a broken away supercon-
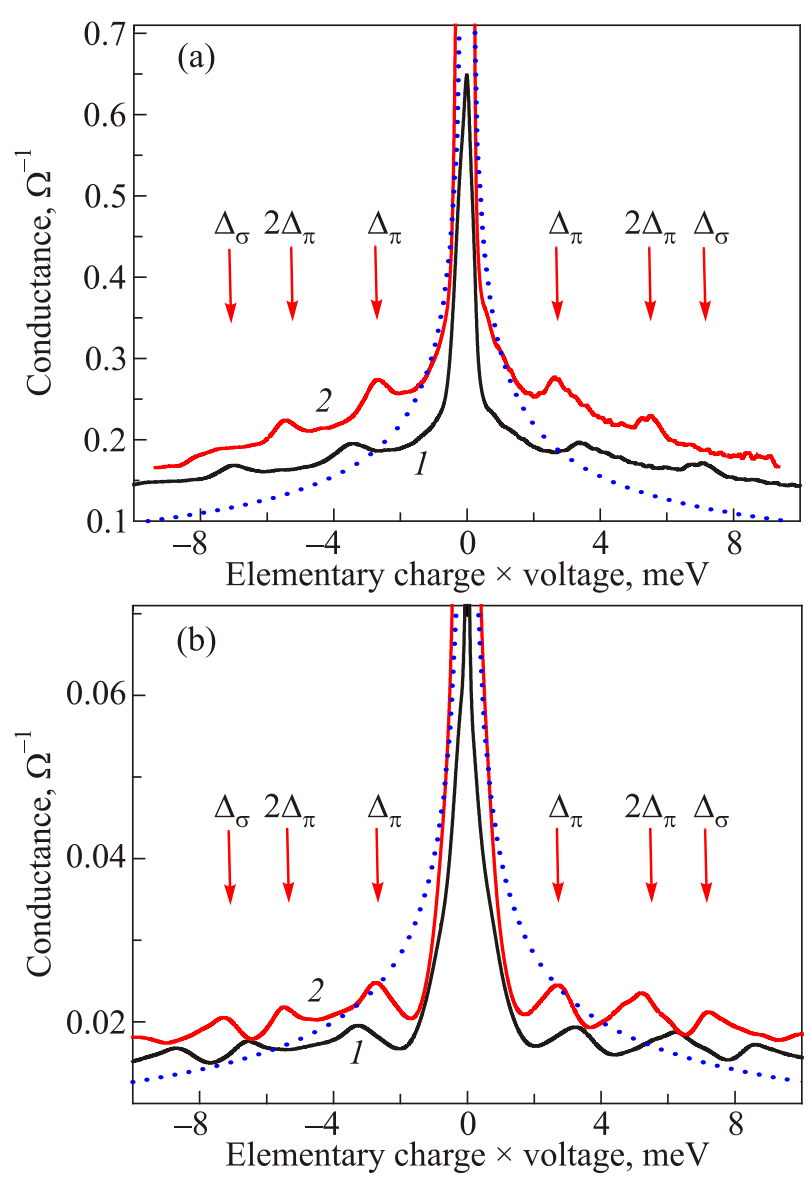

Fig. 3. (Color online) Representative conductance spectra of $\mathrm{Ag} / \mathrm{MgB}_{2}$ junctions at $4.2 \mathrm{~K}$ with two (a) and three (b) lowvoltage features, initial conductance-vs-voltage characteristics (1) and corrected by the influence of the N-c-S contact (2). Dotted curves represent $1 / \sqrt{V}$ low-voltage asymptotic predicted in [13], arrows show positions of $\Delta_{\pi}, 2 \Delta_{\pi}$, and $\Delta_{\sigma}$ singularities expected from calculations [14].

ducting grain and an S-c-S junction between two superconducting pellets. If so, the measured voltage bias equals to $V(I)=V_{N S}(I)+V_{S S}(I)$. In contrast to the paper [8], we assume that we are dealing with two direct $\mathrm{N}-\mathrm{C}-\mathrm{S}$ and $\mathrm{S}-\mathrm{C}-\mathrm{S}$ contacts without any significant barrier between the metallic electrodes. The main characteristics of the first junction are originating from the Andreev reflections at the NS interface were described above. Charge transport in S-c-S contacts includes multiple Andreev scattering processes at two interfaces between superconducting electrodes [13]. It is well known that, they manifest themselves in the so-called "subharmonic gap structure". In a S-c-S junction with similar single-gap superconductors it consists of conductance peaks at voltages $V_{n}= \pm 2 \Delta /(e n), n$ is an integer. In $S_{1}-c-$ $\mathrm{S}_{2}$ asymmetric junctions with dissimilar gap values $\Delta_{1}$ and $\Delta_{2}$ on opposite sides of the scattering region [18] or those with identical two-gap superconductors the number of the singularities strongly increases, in particular, the new ones are $\pm 2 \Delta_{1} /(e n), \pm 2 \Delta_{2} /(e n), \pm 2\left(\Delta_{1}+\Delta_{2}\right) /(e(2 n+1))$, etc. Among them, the most pronounced processes are those 
involving one Andreev reflection which result in conductance peaks at biases $\Delta_{1} / e$ and $\Delta_{2} / e$ (see Fig. 4 in [18]). For voltages much less than the superconducting gaps the dc differential conductance $d I / d V$ of the S-c-S junction was predicted to diverge as $1 / \sqrt{|V|}$ [13]. This singularity is a hallmark of the increased number of Andreev reflections at very small biases before a charge may enter one of the superconducting electrodes. Of course, the effect will be suppressed by any mechanism of inelastic scattering. Nevertheless, in junctions shorter than inelastic scattering length, there should be a voltage range where the conductance follows the $1 / \sqrt{|V|}$ behavior, i.e., goes to infinity at $V \rightarrow 0$.

If so, then the finite junction conductance at zero bias should be completely determined by that of the $\mathrm{N}-\mathrm{C}-\mathrm{S}$ contact. It allows us to estimate the ratio of the normalstate resistances of N-c-S and S-c-S contacts $R_{N S} / R_{S S}$ which was in almost all cases $\leq 0.5$. Therefore, for the total voltage drop $V \leq 10 \mathrm{mV}$ that on the $\mathrm{N}-\mathrm{c}-\mathrm{S}$ contact was less than $\Delta_{\sigma} / e$, the resistance of the $\mathrm{N}-\mathrm{c}-\mathrm{S}$ contact was constant and equal to $R_{N S} / 2$. At the same time, the resistance of the S-c-S contact is negligibly small [13], allowing to assume that $G(0)=2 / R_{N S}$. Taking that into account, we were able to find the voltage bias on the S-c-S contact $V_{S S}(I)=V(I)-I R_{N S} / 2$ and related conductance value $G_{S S}(I)=\left(1 / G(I)-R_{N S} / 2\right)^{-1}$. In Fig. 3 we demonstrate two examples of such correction procedure (curves 2). We can see that after the correction the singularities become more pronounced, they are shifted to lower biases and the low-voltage asymptotic is consistent with the $1 / \sqrt{|V|}$ dependence. Moreover, positions of the features at about 3 and $7 \mathrm{meV}$ now well correspond to $\Delta_{\pi} / e$ and $2 \Delta_{\pi} / e$ values which, according to the theory [13], are the most pronounced features in the conductance spectra of S-c-S contacts with a disordered inter-electrode region. The position of the local maximum at $9 \mathrm{mV}$ seen in Fig. 3(b) is shifted to the expected value of the large gap $\Delta_{\sigma} / e$. It should be noticed that the gap values are very similar to those observed in STM/STS experiments, e.g., [19], and found for planar tunnel junctions (see, e.g., [20]). We believe that the reproducibility of the zero-bias anomaly and the shift of the gap-like features to correct gap positions give the evidence in favor of our hypothesis about two junctions in series realized in our experiments and show that the main nonlinearities in the measured curves descend from the S-c-S contact formed between two $\mathrm{MgB}_{2}$ grains.

A very rare observation of the larger gap $\Delta_{\sigma}$ is actually expected and can be explained by the fact that it requires a strictly defined current direction. Indeed, the total quasiparticle current across the $\mathrm{MgB}_{2}$-based junction is a sum of partial contributions from two electronic bands with a weighing factor $\omega_{p}^{2}[6]$ where $\omega_{p}$ is the plasma frequency for the corresponding band. Due to the smallness of $\omega_{p}^{\sigma}$ in the $c$-direction $\left.\left(\omega_{p}^{\sigma} / \omega_{p}^{\pi}\right)^{2}=0.01[14]\right)$, the charge transport in the $c$-direction of the $\mathrm{MgB}_{2}$ crystal lattice is only determined by the $\pi$-band. But in the crystallographic $a-b$ plane direction contributions from the two bands are comparable since in this case $\left(\omega_{p}^{\sigma} / \omega_{p}^{\pi}\right)^{2}=0.49$. Thus, to observe the larger gap $\Delta_{\sigma}$ we need contacts oriented roughly in the $a-b$ plane while the smaller gap $\Delta_{\pi}$ should be observable for all transport directions. Since the orientation of superconducting grains in $\mathrm{S}-\mathrm{C}-\mathrm{S}$ junctions was uncontrollable in our experiment, the number of the junctions which reveal the feature at $\Delta_{\sigma} / e$ was very small.

To estimate correct peak positions, we have differentiated numerically the $G(V)$ curves and found downwardgoing zero-crossings which correspond to the real maxima. After averaging over eight samples, for the smaller gap, we got $\Delta_{\pi}=(2.78 \pm 0.23) \mathrm{meV}$ whereas, for the larger gap, the mean value for two junctions clearly exhibiting it was $\Delta_{\sigma}=(7.32 \pm 0.09) \mathrm{meV}$.

\section{Conductance spectra at the pressure of $1.5 \mathrm{GPa}$}

Soon after the discovery of superconductivity in $\mathrm{MgB}_{2}$, several groups reported decrease in the critical temperature under high pressure, but the rate of the decrease varied considerably from -1.6 to $-1.9 \mathrm{~K} / \mathrm{GPa}$ in piston-cylinder studies with Fluorinert pressure medium and from $-1.36 \mathrm{~K} / \mathrm{GPa}$ in hydrostatic experiments with a mixture of daphne and kerosene oils to $-0.6 \mathrm{~K} / \mathrm{GPa}$ in quasi-hydrostatic measurements with solid steatite pressure medium (see related references in [9]). Recently, the value $d T_{C} / d P=-1.1 \mathrm{~K} / \mathrm{GPa}$ was suggested to be the intrinsic value for magnesium diboride under hydrostatic pressure [10]. In the preprint [21], a correlation between $T_{\mathrm{C}}$ and the derivative $d T_{C} / d P$ was found: for $T_{C}=39.2 \mathrm{~K}$ and $37.5 \mathrm{~K} d T_{C} / d P \approx-1.07 \mathrm{~K} / \mathrm{GPa}$ and $\approx-1,45 \mathrm{~K} / \mathrm{GPa}$, respectively. As was mentioned above, in our samples the onset temperature as well as a midpoint one decreased with the pressure at a rate of the order of $-1 \mathrm{~K} / \mathrm{GPa}$ (Fig. 1). It can be considered as an additional argument proving that the comparatively wide resistive transition originates from weak links between magnesium diboride grains each of which has a transition temperature close to optimum for a given compound.

After applying the hydrostatic pressure $P$ of $1.5 \mathrm{GPa}$ to the junction formed by a silver tip and a polycrystalline $\mathrm{MgB}_{2}$ sample, the gap-feature positions in conductance-vsvoltage curves have changed. It can be clearly seen from Fig. 4 where we demonstrate how the corrected conductance spectrum shown in Fig. 3(b), curve 2 is modified under the pressure. To find correct positions of the gap peaks and the compression effect on them, we should extract the background in the two curves which was strongly modified at $P=1.5 \mathrm{GPa}$. It was represented by broken-line segments connected the local minimums around corresponding peaks in the conductance spectra (the dashed lines in the main panel in Fig. 4). Results of the peaks extraction are shown as insets in Fig. 4. 


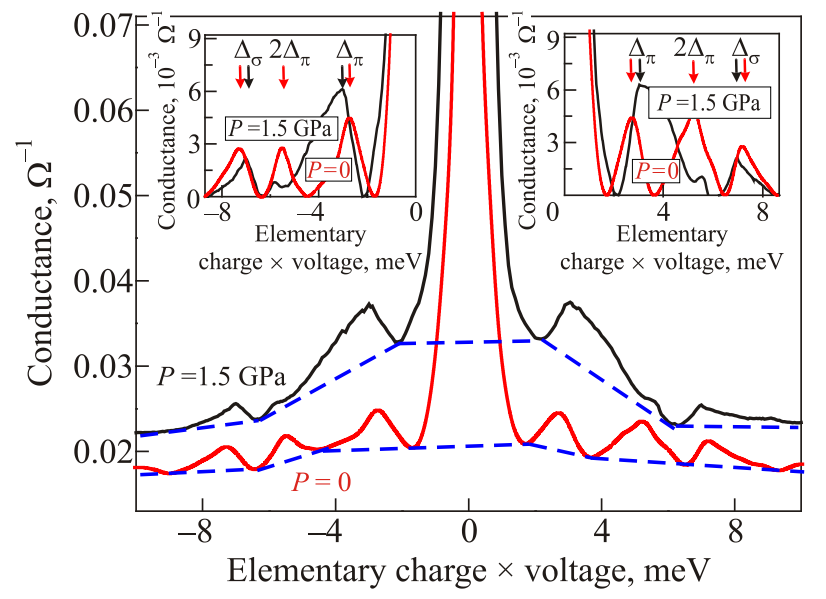

Fig. 4. (Color online) Pressure effect on the conductance spectra of the $\mathrm{Ag} / \mathrm{MgB}_{2}$ junction shown in Fig. 3(b) at $4.2 \mathrm{~K}$, both curves are the result of the correction for ambient pressure and $P=1.5 \mathrm{GPa}$. Left and right insets show results of the background (the dashed lines) extraction at negative and positive voltages, respectively. The arrows in the insets show voltage positions of $\Delta_{\pi}$ and $\Delta_{\sigma}$ features in the corrected characteristics for $P=0$ and $P=1.5 \mathrm{GPa}$.

Notice that from the theoretical point of view there are two factors influencing the energy gap values in the twoband/two-gap model, intraband and interband couplings. Due to the pressure-induced growth in related phonon frequencies, intraband coupling decreases in each band. At the same time, interband coupling is expected to increase when the sample volume is reduced. For the larger gap, the two factors should act in the same direction and, hence, the gap $\Delta_{\sigma}$ should decrease at a rate of the order of the critical temperature. For the smaller gap $\Delta_{\pi}$, the effect of two couplings is opposite and the question which of the two factors is dominant can be solved only by numerical calculations.

Concerning the variation of the critical temperature $T_{C}$ with pressure, there is as yet quite a few reports using realistic models of magnesium diboride (see, as an example, atomistic simulations in [22]). Speaking about theoretical analysis of the pressure effect on the energy-gap structure, there are up to our knowledge only two publications [10] and [23]. The calculations in the work [23] were based on an s-wave, two-band Eliashberg approach reduced to four coupled integral equations for two gaps and two renormalization functions. The author [23] suggested that, in the first approximation, the normal density of states at the Fermi level does not change with pressure. In this way, the variation of the electron-phonon coupling constant $\lambda_{\pi \pi}$ and that of the interband scattering is zero and the $\Delta_{\pi}$ value is almost constant. The second paper [10] utilized fully anisotropic Eliashberg equations, more suitable for analyzing the pressure effect on the low-temperature superconducting characteristics. It was found that the multiple-gap nature of magnesium diboride persists at high pressures, both average values of $\Delta(\mathbf{k})$ on $\sigma$ - and $\pi$-Fermi surfaces decrease substantially, and this effect is especially strong for the smaller gap.

In contrast to theoretical expectations [10,23], our experiment demonstrates an increase in the smaller gap whereas the larger gap decreases with the rate of $d \Delta_{\sigma} / d P=-0.16 \mathrm{meV} / \mathrm{GPa}$ which agrees with that of $-(0.13-0.17) \mathrm{meV} / \mathrm{GPa}$ predicted in the papers $[10,23]$. Note that the feature at $V=2 \Delta_{\pi} / e$ is not well resolved due to the change of the background conductance-vs-voltage dependence under pressure.

\section{Conclusions}

We have performed point-contact measurements on polycrystalline samples of magnesium diboride which exhibited strongly granular internal structure. Contrary to the expectations, we have not observed a conventional Andreev-reflection spectra for $\mathrm{N}-\mathrm{c}-\mathrm{S}$ contacts with a doubled zero-bias conductance comparing to the normal one but rather much higher and steeper peak centered at $V=0$. The finding was interpreted as a formation of two junctions in series: a contact between the normal-metal Ag tip and a grain of magnesium diboride and that between this grain and another $\mathrm{MgB}_{2}$ grain. In contrast to the paper [8], where a similar explanation was proposed for high-resistance tunneling-like sandwiches, we were dealing with metallic $\mathrm{MgB}_{2}-\mathrm{c}-\mathrm{MgB}_{2}$ contacts. It was proven by comparing their conductance-vs-voltage characteristics extracted from the measured curves using an accurate theoretical analysis with those predicted theoretically for short multiple-mode S-c-S contacts with a disordered constriction [13]. After the correction procedure we have usually observed a zerobias $1 / \sqrt{|V|}$-like singularity of the dc differential conductance for voltages smaller than the lower superconducting gap $\Delta_{\pi}$ and two local maxima roughly at $|V|=\Delta_{\pi} / e$ and $|V|=2 \Delta_{\pi} / e$. A few samples have exhibited an additional feature which after the correction procedure was located approximately at $|V|=\Delta_{\sigma} / e$ corresponding to the larger gap.

It should be stressed that it is not the first observation of steep zero-bias peaks with $G(0)>2 G_{N}$ in point contacts of a normal counter-electrode with a superconductor. In particular, they have been observed for iron pnictide single crystals [24] and thin layers [25] as well as in $\mathrm{Fe}(\mathrm{Se}, \mathrm{Te})$ films [26]. It can be that our interpretation of the anomalous zero-bias peaks is valid in these cases as well. Most probably, the suggested junctions-in-series structure appears due to strongly degraded and inhomogeneous surfaces in Fe-based superconductors [27].

The sample with the most pronounced feature shown in Fig. 3(b) was used for the study of the pressure effect on two energy gaps in magnesium diboride, see Fig. 4. The larger gap, as expected [10,23], was found to decrease from $7.25 \mathrm{meV}$ to $7.01 \mathrm{meV}$ while the smaller gap was surprisingly going up from $2.69 \mathrm{meV}$ to $3.07 \mathrm{meV}$ (the gap 
values at $P=0$ and $P=1.5 \mathrm{GPa}$ were obtained by averaging the positions of the maxima in the corrected curves for positive and negative voltage biases). In principle, it could result from the incoherent interband scattering caused by non-magnetic defects which would increase under compression. But $\Delta_{\pi}$ and $\Delta_{\sigma}$ values extracted from our experimental curves well agree with those expected for pure $\mathrm{MgB}_{2}$. We may conclude that the incoherent scattering by inhomogeneities was not important at ambient pressure and, most probably, remained so under the compression of 1.5 GPa. To our opinion, the contradiction with the theory $[10,23]$ clearly indicates that the pressure effect of the interband scattering in clean $\mathrm{MgB}_{2}$ samples has been underestimated. The second conclusion is that, in any case, the separable anisotropy model discussed in the introduction is not able to describe the positive derivative $d \Delta_{\pi} / d P$ since it predicts decrease of all gap values due to increase in phonon frequencies.

\section{Acknowledgments}

This work was performed within the German-Ukrainian projects SE 664/18-1 supported by Deutsche Forschungsgemeinschaft (DFG). S. Schmidt thanks the Landesgraduiertenförderung Thüringen for financial support.

1. X.X. Xi, Rep. Prog. Phys. 71, 116501 (2008).

2. I.I. Mazin and V.P. Antropov, Physica C 385, 49 (2003).

3. A.A. Golubov, J. Kortus, O.V. Dolgov, O. Jepsen, Y. Kong, O.K. Andersen, B.J. Gibson, K. Ahn, and R.K. Kremer, J. Phys.: Condens. Matter 14, 1353 (2002).

4. M. Zehetmayer, Supercond. Sci. Technol. 26, 043001 (2013).

5. D. Markowitz and L.P. Kadanoff, Phys. Rev. 131, 563 (1963).

6. I.I. Mazin, Phys. Rev. Lett. 83, 1427 (1999).

7. C. Grimaldi, Phys. Rev. B 89, 214201 (2014).

8. F. Giubileo, F. Bobba, M. Aprili, S. Piano, A. Scarfato, and A.M. Cucolo, Phys. Rev. B 72, 174518 (2005).

9. J.S. Schilling, in: Frontiers of High Pressure Research II. Application of High Pressure to Low-Dimensional Novel Electronic Materials, NATO Science Series, Springer (2001), vol. 48, p. 345.
10. H.J. Choi, S.G. Louie, and M.L. Cohen, Phys. Rev. B 79, 094518 (2009).

11. V.M. Svistunov, V.Yu. Tarenkov, A.I. D’yachenko, O.I. Chernyak, and R. Aoki, Physica C 303, 177 (1998).

12. V.M. Svistunov, V.Yu. Tarenkov, A.I. D’yachenko, O.I. Chernyak, and R. Aoki, Physica C 314, 205 (1999).

13. A. Bardas and D.V. Averin, Phys. Rev. B 56, 8518(R) (1997).

14. A. Brinkman, A.A. Golubov, H. Rogalla, O.V. Dolgov, J. Kortus, Y. Kong, O. Jepsen, and O.K. Andersen, Phys. Rev. B 65, 180517(R) (2002).

15. D. Daghero and R.S. Gonnelli, Supercond. Sci. Technol. 23, 043001 (2010).

16. J.M. Doval, J. Maza, C. Torron, J.A. Veira, M. Tello, and F. Vidal, Adv. Sci. Tech. 95, 202 (2014).

17. P. Martinez-Samper, J.G. Rodrigo, G. Rubio-Bollinger, H. Suderow, S. Vieira, S. Lee, and S. Tajima, Physica C 385, 233 (2002).

18. M. Ternes, W.-D. Schneider, J.-C. Cuevas, C.P. Lutz, C.F. Hirjibehedin, and A.J. Heinrich, Phys. Rev. B 74, 132501 (2006).

19. M. Iavarone, G. Karapetrov, A.E. Koshelev, W.K. Kwok, G.W. Crabtree, D.G. Hinks, W.N. Kang, E.-M. Choi, H.J. Kim, H.-J. Kim, and S.I. Lee, Phys. Rev. Lett. 89, 187002 (2002).

20. K. Chen, Y. Cui, Q. Li, C.G. Zhuang, Z.-K. Liu, and X.X. Xi, Appl. Phys. Lett. 93, 012502 (2008).

21. B. Lorenz, R.L. Meng, and C.W. Chu, cond-mat/0104303 (2001), unpublished.

22. C. Zhang and X. Zhang, Comput. Mater. Sci. 50, 1097 (2011).

23. G.A. Ummarino, Physica C 423, 96 (2005).

24. X. Lu, W.K. Park, H.Q. Yuan, G.F. Chen, G.L. Luo, N.L. Wang, A.S. Sefat, M.A. McGuire, R. Jin, B.C. Sales, D. Mandrus, J. Gillett, S.E. Sebastian, and L.H. Greene, Supercond. Sci. Technol. 23, 054009 (2010).

25. S. Döring, S. Schmidt, F. Schmidl, V. Tympel, S. Haindl, F. Kurth, K. Iida, L. Mönch, B. Holzapfel, and P. Seidel, Physica C 478, 15 (2012).

26. Y.T. Shen, Y.S. Li, K.C. Lin, M.K. Wu, and C.C. Chi, J. Appl. Phys. 114, 123912 (2013).

27. T. Plecenik, M. Gregor, R. Sobota, M. Truchly, L. Satrapinskyy, F. Kurth, B. Holzapfel, K. Iida, P. Kus, and A. Plecenik, Appl. Phys. Lett. 103, 052601 (2013). 